\title{
Competencias Matemáticas Desarrolladas en Ambientes Virtuales de Aprendizaje: el Caso de MOODLE
}

\author{
Martha L. García(1) y Alma A. Benítez ${ }^{(2)}$ \\ (1) Instituto Politécnico Nacional, ESIME-ZACATENCO, Avenida Instituto Politécnico Nacional, \\ S/N, Col. Lindavista, CP 07738, México D.F.-México (e-mail: martha.garcia@gmail.com) \\ (2) Instituto Politécnico Nacional, CECyT 11. Wilfrido Massieu, Av. de los Maestros 217, \\ Col. Casco de Santo Tomás,. CP 11340, México D. F.-México (e-mail: abenitez@ipn.mx)
}

Recibido Feb. 18, 2011; Aceptado Mar. 17, 2011; Versión final recibida Abr. 04, 2011

\begin{abstract}
Resumen
Este trabajo tiene como objetivo documentar y analizar los tipos de razonamiento que emergen en los estudiantes cuando resuelven problemas de matemáticas e interactúan en un ambiente elearning. Como se ha documentado en numerosas investigaciones, la aparición de diversas tecnologías digitales ha modificado las competencias que ahora requieren los profesionales. Ante este panorama, las instituciones educativas buscan desarrollar en los estudiantes tales competencias, e integran en los procesos de enseñanza aprendizaje el uso de tecnologías digitales. Las matemáticas promueven aquellas competencias relacionadas con el análisis, el razonamiento, y la resolución de problemas. En el trabajo que aquí se reporta, se usó una metodología de tipo cualitativo, y se diseñó una secuencia de ocho actividades. Los resultados muestran dos tipos de razonamiento en el trabajo de los estudiantes y permiten definir las competencias relacionadas con el uso de tecnología que requieren los estudiantes para trabajar en un ambiente virtual de aprendizaje.
\end{abstract}

Palabras clave: competencias, matemáticas, e-learning, razonamiento y representaciones

\section{Mathematical competencies developed in virtual environments of learning: the case of MOODLE}

\begin{abstract}
In this paper the objective is to document and analyze types of students reasoning they developed when working with a set of mathematical problems in the environment e-learning. It has been documented in many researches the emergence of new digital technologies, as a consequence nowadays professionals need to develop new competencies. Educational institutions look for to develop these students' skills and integrate digital technologies into the teaching and learning processes. Mathematics promotes these competencies related to analysis, reasoning and solving problem. In this study researches used a qualitative methodology and designed a sequence of eight activities. The findings obtained make it possible to identify two distinct types of reasoning in students' work as well as competences that individuals need to develop when they use a virtual environment of learning.
\end{abstract}

Keywords: mathematics, competencies, e-learning, reasoning and representations 


\section{INTRODUCCIÓN}

La vida moderna se caracteriza por un creciente uso de los medios tecnológicos, por la digitalización de los datos y por el manejo de una gran cantidad de información. Con este panorama del mundo, surgen nuevas demandas en la formación de las personas quienes ahora deben desarrollar competencias que les permitan acceder a la información, seleccionarla y difundirla desde cualquier medio y desde cualquier parte del mundo. Las tecnologías posibilitan el acceso a una gran cantidad de información en diferentes áreas de conocimiento. Lo anterior ha motivado la reflexión de los dirigentes de los sistemas educativos en distintos países, ya que se considera que la preparación de los estudiantes, incide en el papel que desempeñarán como ciudadanos y es también un indicador del desarrollo de una sociedad (Rico 2007). Para dar respuesta a las demandas actuales se recomienda incluir en la formación escolar de los individuos, el desarrollo de competencias claves que les permitan resolver problemas en un contexto particular, poniendo en juego recursos psicosociales que incluyen habilidades y actitudes. Dentro de estas competencias se incluyen: a) la capacidad de análisis y síntesis; b) la capacidad de aprender; c) la habilidad para resolver problemas; d) la capacidad de aplicar el conocimiento; e) la habilidad para manejar tecnologías digitales; f) las destrezas para manejar la información y g) la capacidad de trabajar autónomamente y en grupo. Estas competencias se pueden vincular con todas las áreas de conocimiento, en particular con el estudio de las matemáticas, que es la disciplina en la que se enmarca el presente trabajo. Por otra parte, las competencias matemáticas se refieren a la capacidad de un individuo para identificar y entender el papel que tienen las matemáticas en el mundo, hacer juicios fundamentados y emplear las matemáticas en aquellos momentos en que se presenten necesidades para su vida individual como ciudadano constructivo, comprometido y reflexivo (ISEI-IVEI, 2004). De acuerdo con los resultados del estudio PISA de 2003, el conocimiento de las matemáticas implica el desarrollo de las siguientes competencias: a) pensar y razonar; b) argumentar; c) comunicar; d) modelar; e) plantear y resolver problemas; f) representar y, g) utilizar el lenguaje simbólico, formal, técnico y las operaciones definidas en este lenguaje (ISEI-IVEI, 2004). En publicaciones más recientes se enfatiza que las matemáticas del bachillerato deben contribuir para que los estudiantes desarrollen competencias que les permitan percibir esta disciplina: como un forma de entender e interpretar un fenómeno y no como una secuencia de algoritmos para ser memorizados y aplicados (ICAS, 2010); como una forma de identificar patrones, realizar conjeturas y verificarlas y como una forma de comunicar su conocimientos de las matemáticas a sus compañeros y profesores utilizando el lenguaje formal y el escrito (ICAS, 2010; Proenza y Leyva, 2006).

El desarrollo de alguna de las competencias anteriores ya es una tarea complicada, si además es necesario que integren tecnologías de la información y comunicación (TIC) a su práctica escolar se agregan dificultades adicionales relacionadas con su uso. Es conveniente tomar en cuenta que para integrar alguna tecnología digital, los estudiantes deberán desarrollar, competencias relacionadas con el manejo de las TIC, para que su integración contribuya al logro de los aprendizajes propuestos (García, 2009). Con el propósito de desarrollar algunas competencias matemáticas incorporando las TIC, se llevó a cabo un proyecto de investigación desarrollado en el IPN en el que participaron estudiantes de ingeniería. En este documento se reportan los resultados derivados del proyecto de investigación relacionados con los tipos de razonamiento que se identificaron en los estudiantes cuando trabajaron en una actividad matemática y en un ambiente virtual de aprendizaje.

\section{ELEMENTOS TEÓRICOS}

La Organización para la Cooperación y el Desarrollo Económico (OECD) realizó un proyecto para evaluar nuevos dominios de competencias (OECD, 2005). En el proyecto se enfatiza que una competencia es mucho más que un conocimiento o habilidad; implica poner en juego demandas complejas con el manejo de recursos sicosociales (que incluyen actitudes y valores) en un contexto particular. En los resultados del proyecto se establecen competencias claves en tres categorías: 
Categoría 1: En esta categoría se incluyen las competencias que los individuos requieren para utilizar un amplio rango de herramientas y para actuar efectivamente con el ambiente; unas físicas como las TIC y otras socioculturales como el lenguaje, Los individuos necesita familiarizarse con las herramientas, para entender en qué forma una herramienta modifica su interacción con el mundo y cómo puede ser empleada para realizar diferentes tareas.

En esta categoría podemos encontrar las siguientes competencias:

Competencia para usar el lenguaje y textos interactivamente.

Competencia para usar conocimiento e información interactivamente.

Competencia para emplear la tecnología interactivamente.

Categoría 2: En esta categoría se incluyen las competencias necesarias para que los individuos deben desarrollar la habilidad para comunicarse con otros y poder interactuar con grupos heterogéneos. Aquí se incluyen las habilidades necesarias para vivir y trabajar con otros, habilidades sociales, e interculturales.

En esta categoría encontramos las siguientes competencias:

Competencia para relacionarse con otros.

Competencia para cooperar.

Competencia para manejar y resolver conflictos.

Categoría 3: En esta categoría se incluyen las competencias necesarias para que los individuos manejen con responsabilidad su vida, se adapten al contexto social y actúen en forma autónoma. Para lograr la autonomía es necesario formar en los individuos una conciencia para el futuro del medio ambiente, de las dinámicas sociales y de los roles que juegan los individuos en una sociedad.

En esta categoría podemos encontrar las siguientes competencias:

Competencia para aportar lo que a uno le corresponde.

Competencia para desarrollar planes de vida y proyectos personales.

Competencia para hacer valer los derechos, intereses, límites y necesidades.

Para medir el desarrollo de las competencias clave en un individuo es necesario construir perfiles de competencias, asumiendo: a) que cuando un sujeto trabaja en un contexto emplea un conjunto de competencias y; b) que las evaluaciones de competencias deben incorporar el uso de las TIC para que se consideren instrumentos de prueba interactivos.

\section{Competencias Matemáticas}

En el estudio de las matemáticas el término competencia matemática se refiere a las capacidades de los estudiantes para analizar, razonar y comunicar eficazmente el proceso de resolución de problemas matemáticos que se presenten en una variedad situaciones. (INECSE, 2005).

El proceso de resolución de un problema incluye diferentes fases entre las que se encuentran: a) Identificar las variables presentes en el problema; b) Representar el problema en forma diferente; c) Establecer relaciones entre las variables del problema; d) Establecer relaciones entre las representaciones empleadas; e) Identificar las matemáticas que pueden ser relevantes para la solución del problema; f) Relacionar el problema con otro más simple; g) Utilizar un modelo matemático para representar el problema; h) Justificar los resultados y i) Comunicar el proceso y la solución.

Este documento se hará referencia las representaciones empleadas por los estudiantes durante la resolución de un problema, así como a la relación que los individuos establecen entre ellas, ya que se considera que el análisis de estos elementos proporciona información del tipo de razonamiento que tienen los estudiantes. En este sentido Parnafes y Disessa, (2004) señalan que el razonamiento de los estudiantes está estrechamente relacionado con la representación que emplean; los mismos autores indican que cada representación resalta u oculta aspectos de un concepto, y que cuando los estudiantes hacen uso de varias representaciones desarrollan una 
comprensión más flexible del mismo. También mencionan que al analizar la relación que los estudiantes establecen entre diferentes representaciones es posible obtener información relacionada con los procesos cognitivos que sigue un individuo durante la resolución de un problema. La información subyacente en cada representación es el punto de partida de distintas inferencias y por tanto de diferentes procesos cognitivos. En el mismo sentido se pueden identificar reportes de investigación en los que se señala la importancia de relacionar conceptos procedimientos y problemas, lo que permite conocer en qué forma los conocimientos derivan en distintas formas de razonar para solucionar los problemas (Proenza y Leyva, 2006).

Por su parte Ainsworth (2006) señala, que los beneficios de utilizar una representación se obtienen después de que un estudiante entiende en qué forma se encuentra codificada la información en la representación, y cuál es su relación con el dominio que representa. Para llevar a cabo esta tarea es posible que los estudiantes requieran seleccionar una representación adecuada para ellos, o construir una, lo que además se convierte en un ejercicio cognitivo.

Las ideas anteriores ponen de manifiesto el complejo proceso cognitivo que se desencadena durante la resolución de un problema. Si durante esta actividad matemática se incorpora alguna tecnología ¿qué competencias adicionales relacionadas con el uso de las TIC tiene que desarrollar un individuo? Al respecto García y Benítez (2009) señalan que la incorporación de las TIC demanda que el profesor replantee su práctica docente para orientar la reflexión de los estudiantes, para lograrlo deberá tomar en cuenta el diseño de actividades apropiadas.

\section{Competencias relacionadas con el uso de las TIC}

El empleo de las TIC ha marcado el inicio del nuevo siglo y ha dado al concepto de alfabetización un nuevo significado. Las nuevas generaciones requieren de habilidades para utilizar las TIC y esto ha dado origen al término de alfabetización digital De acuerdo con Jones y Flannigan (2006) este término se refiere a las habilidades que tiene una persona para realizar tareas en forma efectiva en un ambiente digital, la palabra digital significa que la información es representada en una computadora. La alfabetización digital incluye también, la habilidad para leer e interpretar medios (textos, sonidos e imágenes), para reproducir datos e imágenes en un ambiente digital y para aplicar el conocimiento obtenido de estos ambientes.

Las TIC se consideran herramientas que: a) facilitan la realización de múltiples trabajos en la comunidad educativa: gestión de las instituciones, elaboración de materiales didácticos específicos, como instrumentos de apoyo pedagógico, etc.; b) contribuyen para mejorar los procesos de enseñanza y aprendizaje, c) ofrecen nuevas posibilidades para la innovación educativa, para desarrollar nuevos entornos de aprendizaje virtual, sistemas de tele formación, que contribuyan a superar las limitaciones geográficas que imponen los sistemas educativos presenciales. Se han establecido categorías para evaluar las competencias con que cuenta una persona para realizar tareas en forma efectiva en un ambiente digital; estas competencias se agrupan en once bloques, en este trabajo se hace referencia a seis de ellas: a) Conocimientos de los sistemas informáticos (hardware, redes, software); b) Uso básico del sistema operativo; c) Comunicación interpersonal y trabajo colaborativo en redes; d) Procesamiento de textos; e) Tratamiento de la imagen y f) Actitudes generales ante las TIC (ICEC, 2004).

Un ejemplo de uso de las TIC se encuentra en el E-Learning que es utilizado para ofrecer cursos a distancia. El E-Learning se define como: “...un conjunto de métodos, tecnologías, aplicaciones y servicios orientados a facilitar el aprendizaje a distancia a través de Internet" (Moreira, 2009). Una característica sustancial del E-Learning es que la interacción entre los estudiantes, y la de ellos con el docente es independiente del lugar geográfico en el que se encuentren. La información proporcionada en clase puede ser complementada en un tiempo posterior y los estudiantes pueden modificar sus concepciones iniciales, después de interactuar y discutir en grupos un ejemplo de E-Learning es MOODLE.

MOODLE usa aplicaciones web, los usuarios pueden utilizarlas mediante un servidor web empleando internet. Este software permite la creación, gestión y edición de un curso, y administra 
el ingreso de los alumnos inscritos en él. Un curso MOODLE está conformado por recursos, actividades, chat y foros, las actividades y el foro fueron empleados en la investigación como se explicará en el apartado de Metodología.

\section{METODOLOGÍA}

La metodología que se utilizó para analizar los tipos de razonamiento que tienen los estudiantes cuando trabajan en una actividad matemática en un ambiente virtual de aprendizaje (MOODLE) es de tipo cualitativo. Se diseñó una secuencia de 8 actividades, en este documento se reportan los resultados de una de ellas. En esta actividad se analizó la forma en que los estudiantes relacionan las representaciones verbal y gráfica, para dar sentido a un fenómeno de movimiento. De manera más específica, se recabó información para conocer cómo representan e interpretan, los estudiantes, situaciones que involucran movimiento; si relacionan, en una gráfica distancia-tiempo, la pendiente de una recta con el valor de la velocidad; cómo representan en una gráfica distancia tiempo los cambios en la velocidad en diferentes intervalos de tiempo. En el diseño de la actividad se consideraron dos variables principales: i) El contenido matemático subyacente en la actividad y ii) Las competencias claves y las competencias relacionadas con el uso de tecnología que se espera desarrollen los estudiantes.

\section{El contenido Matemático}

En el contenido matemático se consideraron algunos elementos de los trabajos de Nemirovsky (1992) y Carlson et al. (2002); estos autores enfatizan nociones matemáticas sustanciales que los estudiantes utilizan para describir la variación de una función: i) La identificación de características perceptuales de una gráfica distancia-tiempo. Como comparaciones entre puntos de una curva para determinar en cuáles de ellos, la curva tiene mayor o menor velocidad; ii) La razón de cambio de una función. El cambio en la variable dependiente en relación con cambios en la variable independiente puede analizarse en una gráfica distancia-tiempo; iii) Conformar una idea global de una función, a partir de la variación local de la misma en diferentes intervalos. La identificación de cambios simultáneos en los valores de las variables dependiente e independiente, contribuye para el análisis de situaciones dinámicas y iv) Dar sentido a una relación funcional a partir de la construcción de relaciones entre valores del dominio y el rango de una función (relación entre variables).

\section{Competencias claves y competencias relacionadas con el uso de tecnología}

El análisis del problema que realiza un individuo y las representaciones que emplea dan lugar a distintas formas de razonamiento y a diferentes maneras de comunicar los resultados. El énfasis de esta investigación se centra en el desarrollo de estas competencias matemáticas. En concordancia con las competencias relacionadas con el uso de tecnología, las competencias objeto de interés en esta investigación son: los conocimientos de los sistemas informáticos (hardware, redes, software); el uso básico de internet; la comunicación interpersonal y el trabajo colaborativo; el procesamiento de textos; el tratamiento de imágenes y la actitud general ante las TIC.

\section{Los sujetos y la actividad}

La actividad se desarrolló con tres estudiantes que fueron elegidos de un grupo de 20 estudiantes que cursaban la asignatura de Cálculo Diferencial e Integral del primer semestre de una carrera de ingeniería. La elección de los estudiantes se realizó tomando en cuenta cuatro elementos:

i Que mostraran una forma de trabajo representativa del resto del grupo, y que escribieran sus reportes en forma más detallada.

ii Que tuvieran acceso a una computadora y a internet en sus casas para que esta no fuera una variable que influyera durante la actividad.

iii Que estuvieran inscritos en el campus virtual de la institución (plataforma MOODLE).

iv Que aceptaran participar en la investigación. 
Los estudiantes trabajaron durante seis sesiones, las sesiones se realizaron en una comunidad en MOODLE y los recursos de MOODLE que se emplearon fueron actividades y un foro. Las fuentes para recolectar los datos incluyeron: reportes escritos elaborados en Word y participaciones en el foro de discusión.

\section{Actividad camino al trabajo}

La actividad incluyó el análisis de la siguiente situación: He salido por la mañana al trabajo y camino sin prisa a la parada del autobús. Cuando iba a la mitad del camino, vi el autobús que debía abordar y comencé a correr para alcanzarlo. Corrí tan rápidamente como pude, pero no lo alcancé, así que esperé el siguiente autobús en la parada.

Traza una gráfica distancia-tiempo que represente la situación

¿Cómo puedes representar los momentos en que la persona se movía rápida o lentamente en la gráfica?

¿Cómo puedes representar el momento en que la persona se encuentra esperando el siguiente autobús?

\section{ANÁLISIS DE DATOS Y DISCUSIÓN DE RESULTADOS}

Se analizó cuidadosamente el trabajo de tres estudiantes, los estudiantes manifestaron no tener problema con el majo de MOODLE. Cada participación de los estudiantes en MOODLE, fue retroalimentada por el profesor mediante preguntas, aunque en la medida de lo posible el profesor trató de reducir al mínimo su intervención, para que el proceso de aprendizaje se llevara a cabo en forma natural.

\section{Competencias Matemáticas}

Durante el análisis de los datos se intentó entender el estado de conceptualización de cada estudiante y su relación con las competencias matemáticas mencionadas en el apartado de metodología, poniendo énfasis en los tipos de razonamiento de los estudiantes.

Del trabajo reportado por los estudiantes y de su participación en el foro de discusión, se identificaron dos tipos de razonamiento: a) razonamiento basado en el contexto y b) razonamiento basado en restricciones.

\section{a) Razonamiento basado en el contexto}

Esta forma de razonamiento se caracteriza porque en la descripción de la gráfica el estudiante describe la velocidad que lleva la persona en cada uno de los intervalos de tiempo. Esta forma de razonamiento se identificó en el trabajo de César quién describe una gráfica velocidad tiempo sin reparar en que la gráfica solicitada en la actividad, era una gráfica distancia-tiempo. César no establece una relación entre las variables distancia tiempo que coloca en los ejes y los segmentos de recta que utiliza para representar el movimiento de la persona.

La gráfica distancia-tiempo elaborada por César se presenta en la Fig. 1, César señaló que el primer segmento (segmento horizontal) correspondía a la velocidad que tenía la persona cuando caminaba; para representar el intervalo de tiempo en que la persona empezó a correr, utilizó un segmento inclinado, y para representar el intervalo de tiempo en que la persona disminuyó su velocidad utilizó un segmento inclinado que toca el eje horizontal t.

César: La respuesta se puede proyectar en la gráfica, cuando la persona va caminando, gráficamente se muestra como una recta horizontal y paralela al eje $x$, cuando corre, la gráfica torna una pendiente, sucediendo lo mismo cuando va disminuyendo gradualmente la velocidad, hasta llegar a cero, que es el momento del reposo.

La explicación y la gráfica de César permiten confirmar que no relacionó correctamente las variables distancia y tiempo ya que el segmento de recta horizontal en una gráfica distancia tiempo corresponde a un intervalo de tiempo en el que la persona se encuentra en el mismo lugar. 


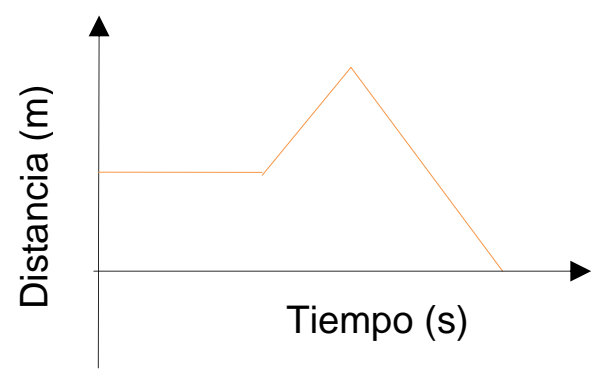

Fig.1: Gráfica distancia-tiempo elaborada por César.

\section{b) Razonamiento basado en restricciones}

Esta forma de razonamiento se caracteriza porque los estudiantes hacen referencia al contexto del problema y también establecen una relación entre las variables que utilizan. Para ejemplificar esta forma de razonamiento, se presentan las gráficas y dos fragmentos de los trabajos de Luis y de Kevin.

La Fig. 2 muestra la gráfica realizada por Luis, en ella representó las diferentes velocidades que lleva la persona cuando se dirige a la parada del camión. Los comentarios escritos por Luis son la evidencia para afirmar que tomó en cuenta el contexto del problema; más aún, en su trabajo se identifica que estableció una relación entre las variables distancia y tiempo en concordancia con la representación gráfica que trazó. Un dato que apoya el comentario anterior, se observa cuando César representa con un segmento de recta horizontal marcado con el número 3 , el intervalo de tiempo en el que la persona se detiene para esperar el camión. También es relevante comentar que Luis utiliza una curva más abierta marcada con el número 2, para representar el intervalo de tiempo en que la persona corre para alcanzar el camión; y una curva más cerrada marcada con el número 4, para representar el intervalo de tiempo en que la persona ya abordó el camión. La segunda curva corresponde a un movimiento con mayor velocidad.

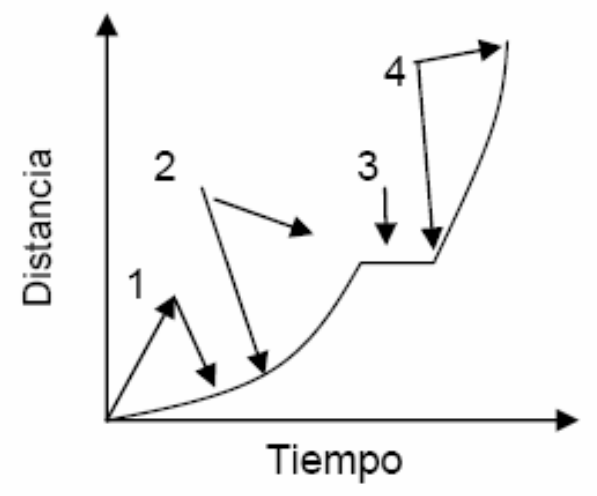

Fig.2: Gráfica distancia-tiempo elaborada por Luis.

Luis: 1. La persona va caminando; 2. La persona empieza a correr; 3. La persona se queda esperando el camión; 4. La persona logra tomar el camión.

En el trabajo de Kevin, que es el tercer estudiante, también se identificó un razonamiento basado en restricciones. La Fig. 3 corresponde a la gráfica de Kevin. Consta de tres segmentos de recta que corresponden a los tres intervalos de tiempo mencionados en el contexto del problema: cuando la persona camina, cuando corre para alcanzar el camión y cuando se detiene en la parada del camión. Kevin representó en forma correcta, con una gráfica distancia tiempo, el movimiento de la persona, su trabajo se complementó con la explicación escrita en la que realizó un análisis más detallado de la situación y estableció en forma precisa una relación entre las variables que utilizó. Kevin indicó que si se considera un mismo intervalo de tiempo, recorrer mayor distancia indica un movimiento más rápido, lo que coincide con el segundo segmento de recta que utilizó para representar esta situación. 


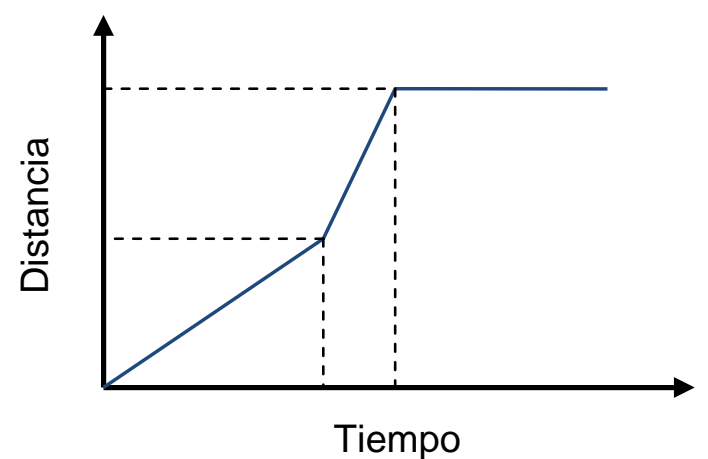

Fig.3: Gráfica distancia-tiempo elaborada por Kevin

Kevin: Si una persona se mueve de forma lenta, la distancia que recorre será menor en un intervalo de tiempo.

Kevin: Si lo hace de forma rápida la distancia recorrida será mayor en ese mismo intervalo de tiempo. En conclusión suponiendo que los intervalos de tiempo son los mismos si la persona se mueve rápidamente cubrirá más distancia que si lo hace lentamente.

Por tanto, en la grafica representé una distancia x y a la mitad de esa distancia la velocidad del tipo aumentó así que la recta se hizo más inclinada, es decir, recorrió la misma distancia pero en menor tiempo.

Luis y Kevin representaron el movimiento de la persona con tres curvas y tres segmentos de recta respectivamente. Las gráficas y la explicación escrita por Luis y Kevin confirman que construyeron una idea global de la función que representa el movimiento que incluye la variación de la misma en diferentes intervalos de tiempo.

\section{Competencias relacionadas con el uso de MOODLE}

De la participación de César, Luis y Kevin en MOODLE, se identificaron las siguientes competencias.

1. Conocimientos de los sistemas informáticos (hardware, redes, software).

La Fig. 4 muestra la página de acceso a las actividades en MOODLE, la figura 5 la interacción de César, Luis y Kevin en la plataforma MOODLE. Los estudiantes manifestaron familiaridad con el manejo de la plataforma.

En la Fig. 5 se observan las imágenes que colocaron Luis y Kevin para identificarse en la plataforma. En el siguiente fragmento se presenta un comentario escrito por Kevin que apoya la afirmación de que los estudiantes se familiarizaron rápidamente con el uso de MOODLE.

Kevin: Damn!, esto se parece mucho a twiter y a decir verdad, "para pa pa pá... me encanta" (si así como McDonald's).

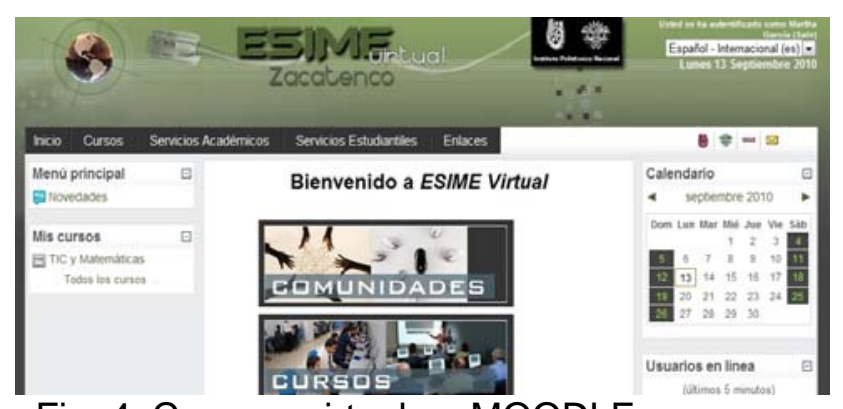

Fig. 4: Campus virtual en MOODLE

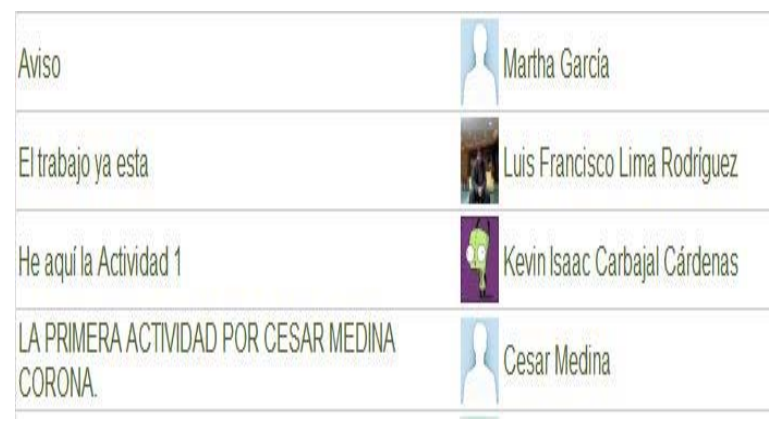

Fig. 5: Trabajo de César, Luis y Kevin en MOODLE 
2. Uso básico del sistema operativo. Las figuras 6 y 7 muestran el trabajo realizado por Luis y Kevin, y apoyan la idea de que los estudiantes manejaron sin problema las carpetas y archivos colocados en la plataforma, también recuperaron el archivo que incluía la actividad para trabajar en ella de manera individual sin problema.

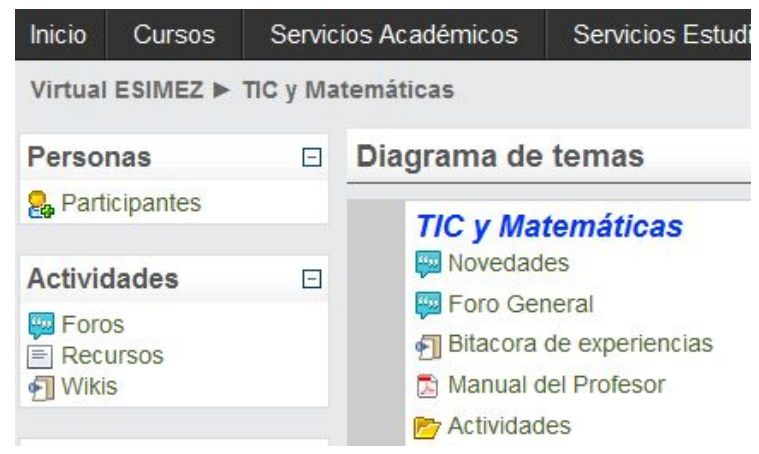

Fig.6: Actividad incluida en la carpeta actividades

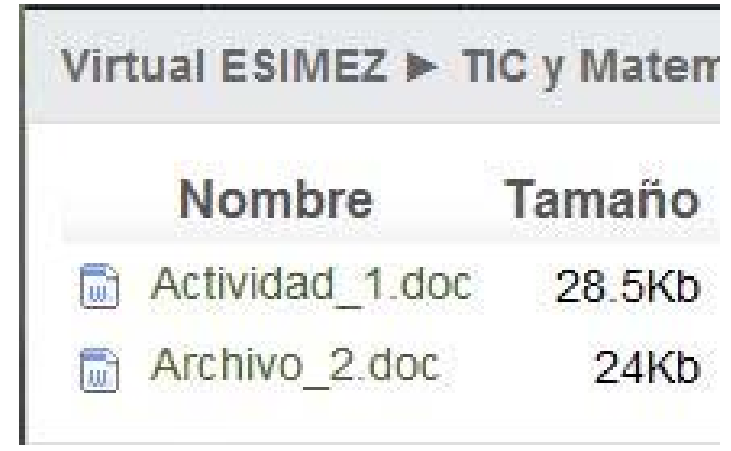

Fig.7: Recuperación del archivo de Word actividad_1.doc

3. Procesamiento de textos. César, Luis y Kevin redactaron en Word un documento para explicar la gráfica que trazaron. En el archivo de Word se identifica que César y Kevin utilizan las opciones para dar formato al texto (incluyendo las opciones de tipo de letra, márgenes, espaciado entre líneas etc.). Luis no utilizó el corrector gramatical de Word y en su reporte se identifican errores gramaticales.

4. Tratamiento de la imagen. El trabajo realizado por César, Kevin y Luis permite inferir que los tres estudiantes utilizaron las funciones básicas de un editor gráfico para hacer dibujos y gráficas en un plano cartesiano.

5. Actitudes generales ante las TIC. La actitud de César para emplear MOODLE fue abierta, entusiasta y participativa; asumió el papel de líder y en todo momento animó a sus compañeros para desarrollar la actividad. De los tres estudiantes fue el que mantuvo más comunicación con el profesor.

César: No tardes Luis, no podemos continuar sin tu apoyo...

César: Hola profesor, estamos en este momento discutiendo de qué gráfica es la correcta... todavía no concluimos, porque evidentemente defendemos nuestro trabajo, sin embargo, sólo un trabajo es correcto... no tardaremos.

La participación de Luis fue moderada, esperó a que César subiera primero su trabajo y comentarios. Kevin fue el que menos participaciones realizó. Sin embargo, como se pudo observar en su reporte, contaba con los conocimientos necesarios para justificar sus respuestas y convencer a sus compañeros de la veracidad de sus comentarios.

6. Comunicación interpersonal y trabajo colaborativo en redes.

Esta competencia está relacionada con el uso responsable de las TIC como medio de comunicación interpersonal en grupos de trabajo, chats y foros. Probablemente por su naturaleza, es la más difícil de desarrollar ya que aún en los ambientes presenciales es difícil la comunicación entre los estudiantes y lograr el trabajo colaborativo.

En relación con esta competencia, en el trabajo de César, Luis y Kevin se identificó que aún cuando trabajaron en grupo, no lo hicieron en el ambiente virtual. César fue el responsable de enviar el trabajo realizado en grupo como lo señala en el siguiente fragmento.

César: Anteriormente cada uno de nosotros (Kevin, Luis y César) le enviamos nuestros trabajos, pero nos dimos cuenta que diferimos en los resultados. Es por ello que le mostramos lo que llegamos como equipo. 
Los comentarios de César y la forma en que presentaron sus reportes, hacen suponer que las discusiones de la actividad se llevaron a cabo en el aula. Un posible factor para que no interactuaran en el campus virtual pudo ser la dificultad de emplear el lenguaje escrito para explicar las gráficas propias y las de sus compañeros.

\section{CONCLUSIONES}

De la evidencia presentada, la discusión y los referentes teóricos expuestos a través del artículo, se pueden obtener las siguientes conclusiones:

1. Se identificaron dos tipos de razonamiento relacionados con el contexto y la representación gráfica que los estudiantes emplean: a) razonamiento basado en el contexto, en el que la gráfica y la explicación escrita del estudiante no se encuentran relacionadas. y, b) razonamiento basado en restricciones, que se caracterizó por una comprensión más completa de la actividad y el establecimiento de relaciones entre las variables del problema, presentes tanto en la gráfica como en la explicación escrita.

2. El diseño de las actividades debe tomar en cuenta las competencias matemáticas que se pretenden desarrollar con la actividad sin menoscabo de las competencias relacionadas con el empleo de cualquier tecnología.

3. El trabajo en una ambiente virtual de aprendizaje como MOODLE requiere que los estudiantes desarrollen nuevas habilidades para efectuar discusiones en grupos, responder por e-mail y participen en discusiones a través de foros,

4. El trabajo de los estudiantes muestra que la interacción colaborativa no se presenta de manera espontánea y debe ser parte de la agenda del profesor.

5. La comunicación entre estudiantes en un ambiente virtual se desarrolla a través de actividades planeadas y dirigidas y es un proceso a largo plazo. Los resultados de las investigaciones en este campo pueden contribuir al desarrollo de las competencias necesarias para la integración de una tecnología en el aprendizaje de cualquier disciplina.

\section{AGRADECIMIENTOS}

Las autoras agradecen el patrocinio otorgado por la Comisión y Fomento a las Actividades Académicas [COFAA-IPN] para realizar y presentar este artículo. Las investigaciones con números de registro 20100459 y 20100678 han sido apoyadas por la SIP del IPN.

\section{REFERENCIAS}

Ainsworth, S., A conceptual framework for considering learning with multiple representations, Learning and Instruction: 16 183-198 (2006).

Carlson, M., Jacobs, S., Coe, E., Larsen, S. y Hsu, E., Applying covariational reasoning while modeling dynamic events: a framework and study, Journal for Research in Mathematics Education: 33(5), 352-378 (2002).

ICEC Instituto Canario de Evaluación y Calidad Educativa, Competencias básicas en las tecnologías de la información y la comunicación (TIC). Evaluación e Investigación Educativa Editores, España, (2004).

García, M. Construcción del concepto de variación con apoyo de una herramienta computacional, Innovación Educativa, Vol. 9, 48, México (2009). 
García, M. y Benítez, A. El papel de las herramientas computacionales y la resolución de problemas en la reflexión de los estudiantes de Matemáticas, Memorias del Segundo Congreso Internacional de Orientación Educativa y Vocacional, 64-71, México 25 a 27 de Marzo (2009).

ICAS Intersegmental Committee of the Academic Senates, Statement of competencies in mathematics expected of entering college students, California State University and University of California, http://icas-ca.org/competencies-in-mathematics (2010).

INECSE Instituto Nacional de Evaluación y Calidad del Sistema Educativo de España, Informe PISA 2003, Pruebas de matemáticas y de solución de problemas, Madrid: Santillana, http://www.gobiernodecanarias.org/educacion/Portal/WebICEC/docs/0809/PISA/pisa2003liberados .pdf (2005).

ISEI-IVEI Instituto Vasco de Evaluación e Investigación Educativa, Primer Informe de la Evaluación PISA 2003, http://www.isei-ivei.net/cast/pub/PISA2003euskadic1.pdf (2004).

Jones, B. y Flannigan, S., Connecting the digital dots: Literacy of the 21st century. Educause Quarterly, No. 2, http://net.educause.edu/ir/library/pdf/eqm0621.pdf (2006).

Moreira, M., Introducción a la Tecnología Educativa, Universidad de la Laguna, España http://webpages.ull.es/users/manarea/ebookte.pdf (2009).

Nemirovsky, R., Students' tendency to assume resemblances between a function and its derivative. Reports-Research/Technical (143), http://edres.org/eric/ED351193.htm. (1992).

OECD Organización para la Cooperación y el Desarrollo Económico, Definition and Selection of Key Competencies: Executive Summary,

http://www.oecd.org/document/17/0,3343,en_2649_39263238_2669073_1_1_1_1,00.html (2005)

Parnafes, O. y Disessa, A., Relations between types of reasoning and computational representations, International Journal of Computers for Mathematical Learning, Kluwer Academic Publishers: Netherlands, 9: 251-280, (2004).

Proenza, Y. y Leyva, L.M., Reflexiones sobre la calidad del aprendizaje y de las competencias matemáticas, Revista Iberoamericana de Educación, (40)6, 1-11, (2006).

Rico, L., La competencia matemática en PISA.PNA, 1(2), 47-66, http://funes.uniandes.edu.co/529/1/RicoL07-2777.PDF (2007). 
